\title{
EPITELIOPATÍA PIGMENTARIA PLACOIDE MULTIFOCAL AGUDA ASOCIADA A PARÁLISIS DEL VI PAR CRANEAL
}

\author{
PIGMENTARY EPITHELIOPATHY MULTIFOCAL ACUTE \\ PLACOID ASSOCIATED WITH PARALYSIS OF VI CRANIAL PAR
}

\author{
GIBELALDE A ${ }^{1}$, BIDAGUREN A ${ }^{1}$, OSTOLAZA JI ${ }^{1}$, CORTÁZAR L ${ }^{1}$, IRIGOYEN C ${ }^{1}$
}

\begin{abstract}
RESUMEN
Caso clínico: Se presenta el caso de un paciente de 24 años con una epiteliopatía multifocal placoide posterior bilateral aguda, confirmada mediante angiografía fluoresceínica. Al mes de seguimiento, el paciente presentó diplopia por paresia del VI par craneal derecho.

Discusión: La epiteliopatía multifocal placoide posterior, es una entidad que se engloba dentro de los síndromes de puntos blancos. Se considera una patología benigna, aunque en ocasiones se asocian alteraciones neurológicas que pueden manifestarse incluso meses después del episodio. Nuestro paciente presentó una paresia del VI. par como complicación neurológica, hecho no descrito en la literatura.
\end{abstract}

Palabras clave: Epiteliopatía multifocal placoide bilateral aguda, parálisis del VI par craneal.

\begin{abstract}
Clinical case: We report the case of a 24-year-old patient who attended our hospital with an acute posterior multifocal placoid pigment epitheliopathy (APMPPE) which was later confirmed by fluorescein angiography. One month after presentation the patient developed a right VI nerve palsy.

Discussion: APMPPE is an acute-onset bilateral inflammatory disease causing impaired vision.

Although it is thought to be benign, neurologic manifestations have been described even months after presentation. There is no previous report of APMPPE associated with VI nerve palsy (Arch Soc Esp Oftalmol 2009; 84: 159-162).
\end{abstract}

Key words: : Acute posterior multifocal placoid pigment epitheliopathy, $\mathrm{VI}^{\text {th }}$ nerve palsy

senta en pacientes jóvenes y adultos de mediana edad, con igual incidencia en ambos sexos. Clínicamente cursa con una disminución de la agudeza visual (AV) que suele ser bilateral y asimétrica. Generalmente es de curso benigno y se resuelve sin

Recibido: 31/5/07. Aceptado: 20/3/09.

Servicio de Oftalmología. Hospital Donostia. San Sebastián. España.

1 Licenciado en Medicina.

Correspondencia:

Ane Gibelalde González

C/. Mimendi, $14,2{ }^{\circ}$ B

20140 Andoain (Guipúzcoa)

España

E-mail: anegibelalde@ hotmail.com 
tratamiento en un plazo de 6 a 12 semanas, dejando áreas de cambios pigmentarios a nivel del epitelio pigmentario. Se presenta el caso de EPPMA bilateral en un paciente de 24 años con manifestaciones neurológicas al mes de seguimiento.

\section{CASO CLÍNICO}

Se presenta el caso de un varón de 24 años de edad, que acude a urgencias por disminución de la AV bilateral y cefalea de dos días de evolución. Como antecedentes personales destacables el paciente refería un cuadro gripal la semana previa tratado con ibuprofeno $600 \mathrm{mg}$. A la exploración la AV del ojo derecho es de bultos y la AV de ojo izquierdo es de 0,05. Las pupilas son isocóricas y normorreactivas y la biomicroscopía del polo anterior es normal. En la oftalmoscopía observamos lesiones confluentes blanco-grisáceas en polo posterior en ambos ojos que afectan a la fóvea (fig. 1). Ante la sospecha de EPPMA, se realizó una angiografía fluoresceínica (AGF), donde se observan áreas de hipofluorescencia marcadas en tiempos precoces (fig. 2) que se convierten en áreas de hiperfluorescencia en tiempos tardíos (fig. 3). La angiografía con verde de indocianina (ICG), muestra áreas de hipofluorescencia en la coroides que corresponden a las lesiones placoides que están en relación con la hipoperfusión coroidea que acompaña a esta patología (fig. 4). La tomografía de coherencia óptica muestra un engrosamiento retiniano con hiperre-

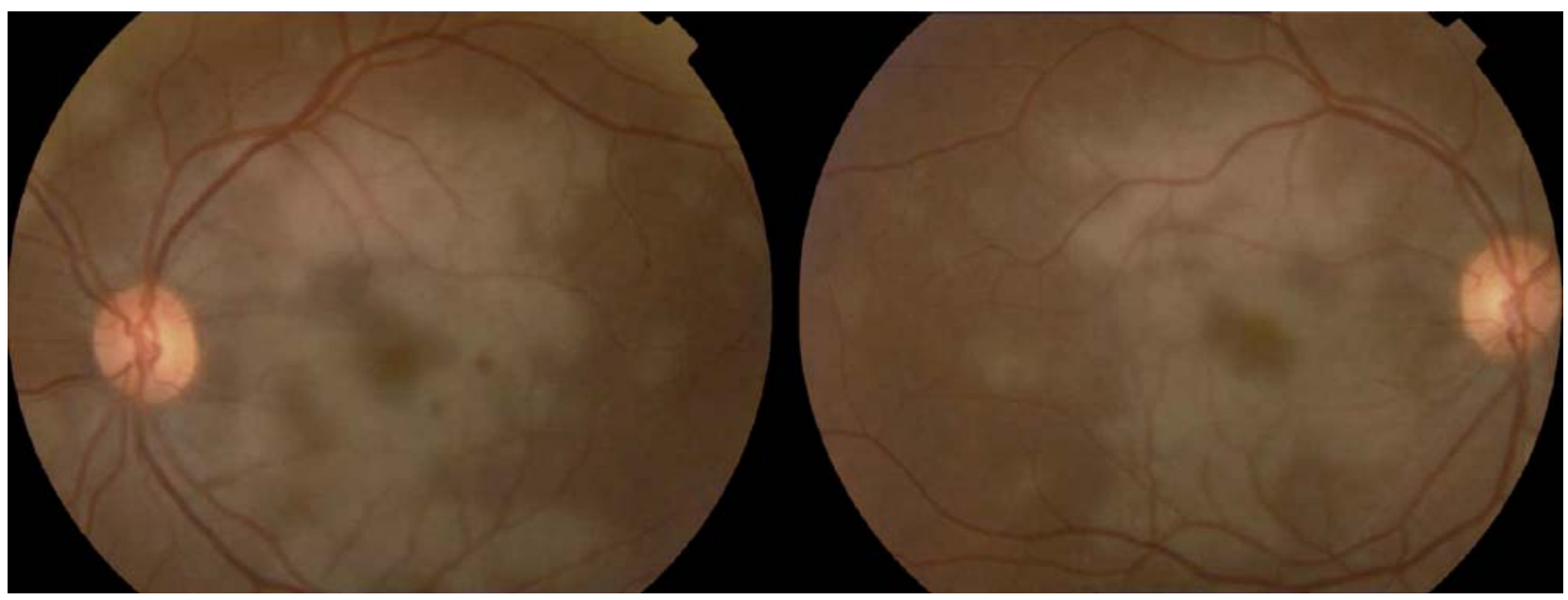

Fig. 1: Oftalmoscopía: Lesiones blanco-grisáceas en polo posterior.

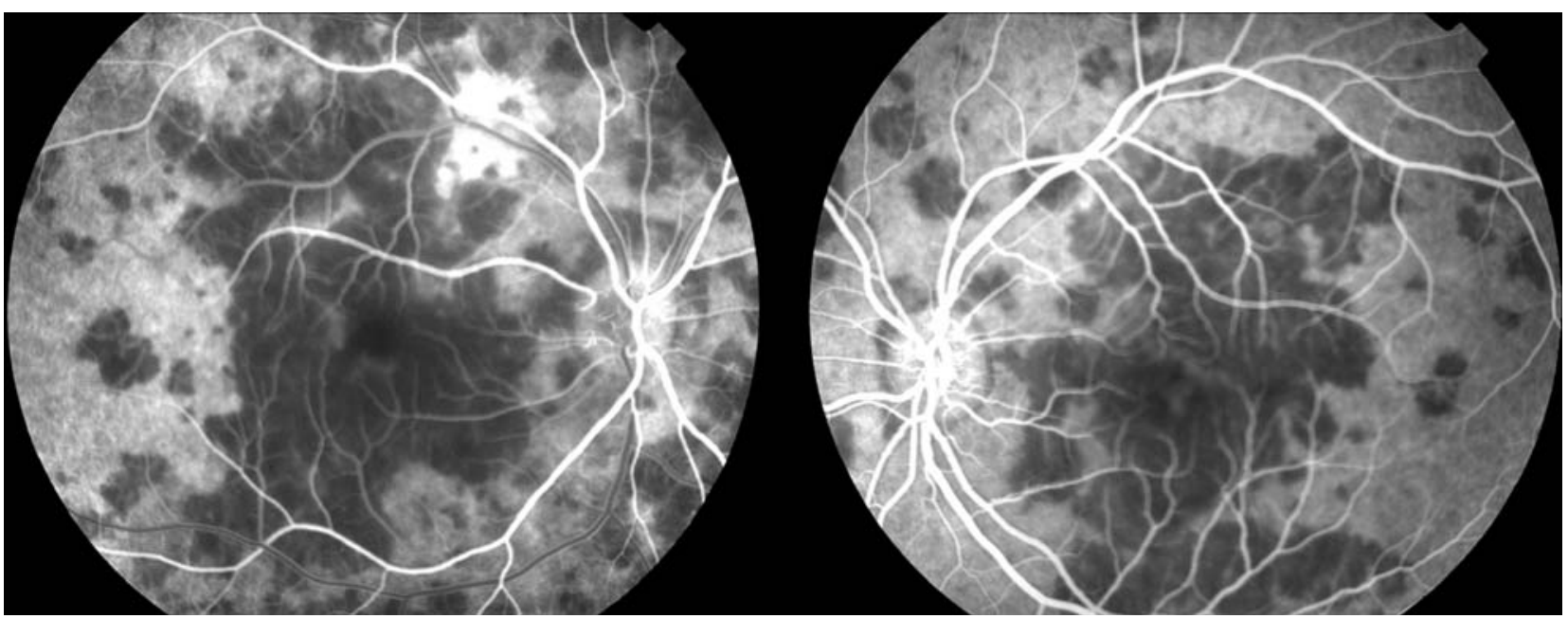

Fig. 2: Angiografía fluoresceínica: áreas hipofluorescentes en tiempos precoces. 


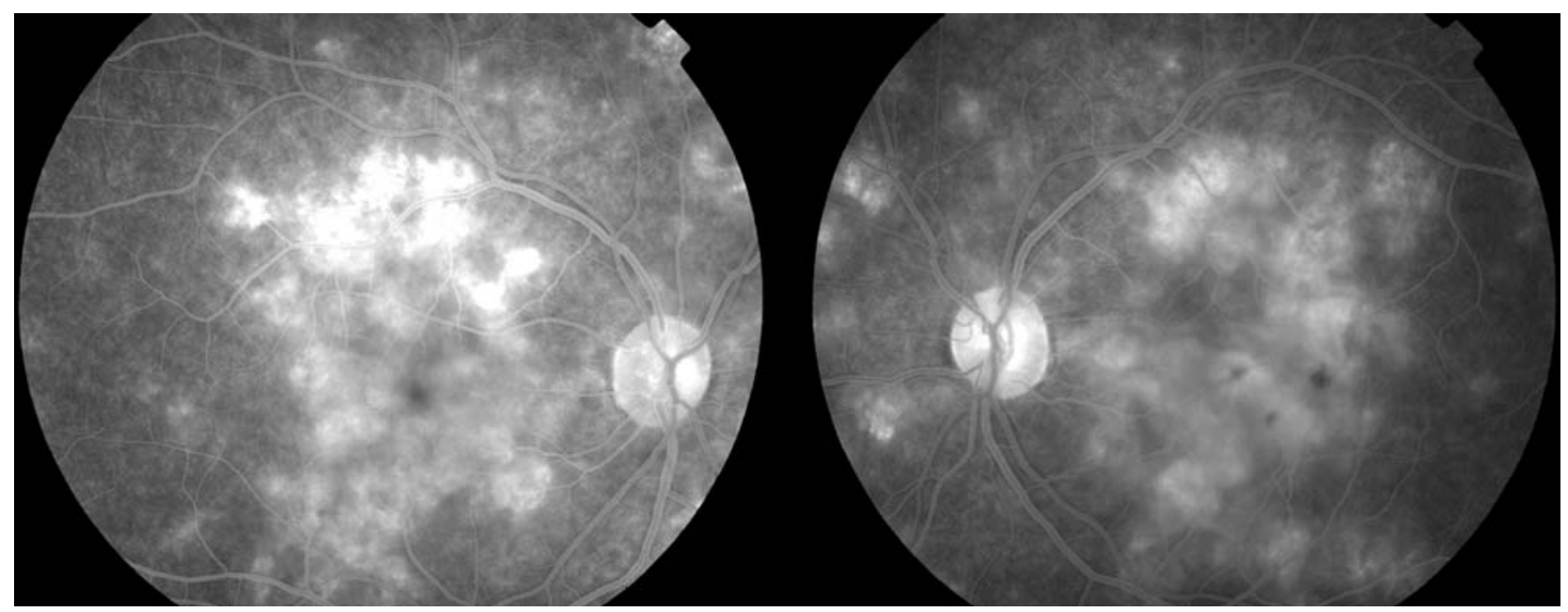

Fig. 3: Angiografía fluoresceínica: zonas de hiperfluorescencia en tiempos tardios.

flectividad a nivel de las capas retinianas externas. Se inició tratamiento corticoideo oral (prednisona $60 \mathrm{mg}$ ) que se prolongó durante una semana.

Por otra parte, el paciente refería una cefalea intensa que mejoró con el tratamiento corticoideo y que reapareció al suspender la medicación por lo tanto ante la sospecha de vasculitis cerebral, que se asocia en ocasiones a este cuadro, se realizó una angiorresonancia magnética nuclear (angioRMN), en la que no se observaban signos de vasculitis.

Se solicitó estudio de histocompatibilidad de HLA B-7 y HLA DR2, que resultó ser positivo.

En cuanto a la evolución, la agudeza visual mejoró paulatinamente pero al mes de seguimiento el paciente refiere diplopía en la visión lejana. A la exploración se observa una parálisis del VI par cra- neal derecho, con leve limitación de la abducción del ojo derecho, hecho que se confirmó con el Test de Hess-Lancaster. Se realizó una inyección de toxina botulínica en el recto medio del ojo derecho y a la semana desapareció la diplopía.

En la actualidad la diplopía han desaparecido y la AV es de 0,7 en ojo derecho y de 0,8 en ojo izquierdo. En la OCT, se demuestra una recuperación de la arquitectura foveal, pero con un aumento de la reflectividad retiniana en ambos ojos.

\section{DISCUSIÓN}

La epiteliopatía multifocal placoide posterior, es una entidad poco frecuente que se engloba dentro

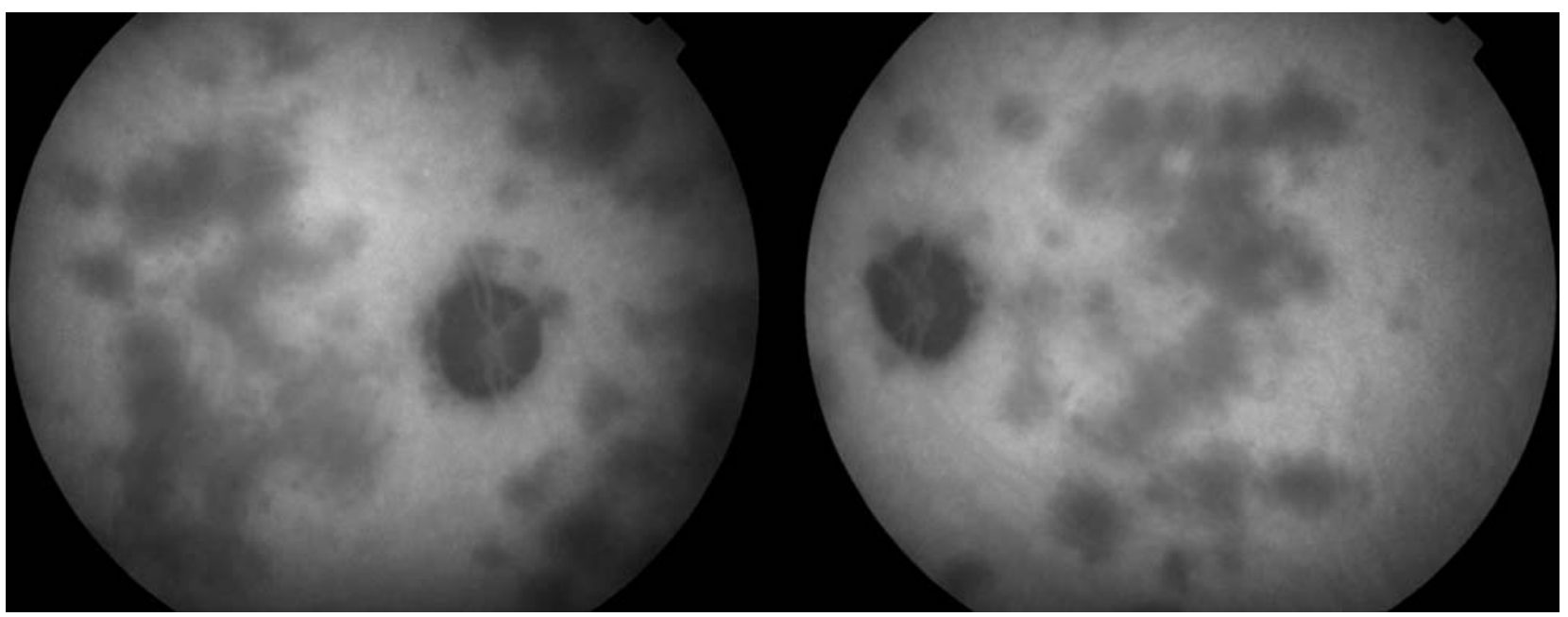

Fig. 4: Angiografía con verde de indocianina: se observan las áreas de hipoperfusión coroidea. 
de los síndromes de puntos blancos. En general se considera una patología benigna por su carácter autoresolutivo, aunque no hay que olvidar que si las lesiones engloban la fóvea, no se consigue una recuperación visual completa. Un 40\% de los casos, refieren un cuadro pseudogripal la semana previa a la disminución de la AV.

La angiografía fluoresceínica, revela la presencia de zonas de hipofluorescencia en tiempos precoces, con zonas hiperfluorescentes en tiempos tardíos. La angiografía por verde de indocianina (ICG) muestra áreas de hipofluorescencia que coinciden con las lesiones placoides, en relación a la hipoperfusión coroidea secundaria a zonas de oclusión vascular (4). La tomografía de coherencia óptica de estos pacientes muestran un engrosamiento retiniano con un aumento de la reflectividad en las capas externas de la retina (5). En nuestro caso, el engrosamiento retiniano ha desaparecido, pero persiste una hiperreflectividad retiniana.

Un porcentaje pequeño de casos se acompañan con manifestaciones neurológicas, que pueden ser más o menos graves. La cefalea está en relación a la asociación de la EPPMA con una meningitis aséptica, que no requiere tratamiento, pero es importante realizar una exploración neurológica adecuada (1). Los pacientes pueden presentar diferentes gra- dos de vasculitis cerebral y meningoencefalitis, que pueden aparecer semanas o meses después de la EPPMA $(1,3)$. Nuestro paciente presentó diplopía al mes de seguimiento que a la exploración resultó ser una parálisis del VI par craneal derecho. Se resolvió favorablemente tras inyección de toxina botulínica en el recto medio del ojo derecho. Esta complicación neurológica no ha sido constatada hasta ahora en la literatura.

\section{BIBLIOGRAFÍA}

1. Çоти S, Verstraeten T, Rinkoff J, Busis N. Neurological manifestations of acute posterior multifocal placoid pigment epitheliopathy. Stroke 1996; 27: 996-1001.

2. Fishman GA, Baskin M, Jednock N. Spinal fluid pleocytosis in acute posterior multifocal placoid pigment epitheliopathy.Ann Ophthalmol 1977; 9: 36-46.

3. Wilson CA, Choromokos EA, Sheppard R. Acute posterior multifocal placoid pigment epitheliopathy and cerebral vasculitis. Arch Ophthalmol 1988; 106: 796-800.

4. Schenider U, Inhoffen W, Gelisken F. Indocyanine green angiography in a case of unilateral recurrent posterior acute multifocal placoid pigment epitheliopathy. Acta Ophthalmol Scand 2003; 81: 72-75.

5. Lim LL, Watzke RC, Lauer AK, Smith JR. Ocular coherence tomography in acute posterior multifocal placoid pigment epitheliopathy. Clin Exp Ophthalmol 2006; 34: 810812. 